\title{
Optical Peaking Enhancement in High-Speed Ring Modulators
}

\section{SUBJECT AREAS: \\ OPTOELECTRONIC \\ DEVICES AND \\ COMPONENTS}

INTEGRATED OPTICS

FIBRE OPTICS AND OPTICAL COMMUNICATIONS

Received

13 August 2014

Accepted

19 August 2014

Published

11 September 2014

Correspondence and requests for materials should be addressed to

J.W. (jwitzens@iph. rwth-aachen.de)

\author{
J. Müller, F. Merget, S. Sharif Azadeh, J. Hauck, S. Romero García, B. Shen \& J. Witzens
}

Integrated Photonics Laboratory, RWTH Aachen, Sommerfeldstr. 14, D-52074 Aachen, Germany.

Ring resonator modulators (RRM) combine extreme compactness, low power consumption and wavelength division multiplexing functionality, making them a frontrunner for addressing the scalability requirements of short distance optical links. To extend data rates beyond the classically assumed bandwidth capability, we derive and experimentally verify closed form equations of the electro-optic response and asymmetric side band generation resulting from inherent transient time dynamics and leverage these to significantly improve device performance. An equivalent circuit description with a commonly used peaking amplifier model allows straightforward assessment of the effect on existing communication system architectures. A small signal analytical expression of peaking in the electro-optic response of RRMs is derived and used to extend the electro-optic bandwidth of the device above $40 \mathrm{GHz}$ as well as to open eye diagrams penalized by intersymbol interference at 32, 40 and $44 \mathrm{Gbps}$. Predicted peaking and asymmetric side band generation are in excellent agreement with experiments.

$\mathrm{T}$ o support fast growing port counts of short distance optical links in data- and high-performance computing centers, integrated electro-optic (E/O) modulators and transceiver subsystems need to be low power and compact in addition to supporting high data rates. $\mathrm{RRMs}^{1}$ and their implementation in silicon technology ${ }^{2}$ combine extreme compactness and low power consumption ${ }^{3}$ with very high modulation speeds $s^{4-6}$ and may thus become an important building block in future interconnect systems. As mass-manufacturable optical interconnect technologies mature and become more affordable, their field of application penetrates to ever smaller architecture levels. After servicing ultra-high-speed links over distances down to a few meters, optical technologies may be applied to even smaller architectural levels within individual servers and supercomputers. Novel architectures such as optically enabled silicon interposers ${ }^{7}$ may enable ultra-high-speed intra-server chip-to-chip connectivity and support emerging applications such as RAM databases or massively parallelized solid-state memories driven by Big Data applications. Silicon Photonics has developed into an integrated E/O technology platform that can address these needs ${ }^{8}$.

Following the initial demonstration of silicon based RRMs with phase shifters based on forward biased PIN junctions $s^{2}$ and improvement of demonstrated data rates with pre-emphasis ${ }^{9}$, implementation with reverse biased $\mathrm{P}(\mathrm{I}) \mathrm{N}$ phase shifters has allowed reaching very high modulation speeds. With dimensions on the order of $10 \mu \mathrm{m}$, RRMs are not only extremely compact relative to non-resonant devices such as travelling wave Mach-Zehnder modulators ${ }^{10,11}$, but also combine modulation with frequency selectivity, thus allowing the realization of wavelength domain multiplexed (WDM) transmitters by cascading multiple RRMs on a single waveguide bus ${ }^{12}$ without requiring additional WDM multiplexers. Suitable compact light sources generating a complete comb of optical carriers with sufficiently narrow linewidth and low relative intensity noise have been realized, e.g., in the form of single section semiconductor comb lasers ${ }^{13,14}$ in which the spectrum of a Fabry-Perot laser is stabilized by means of mode-locking, or in the form of on-chip frequency conversion in monolithically integrated optical parametric oscillators ${ }^{15-17}$. Both classes of light sources have proven to be compatible with multi-channel highspeed communication, the latter supporting even demanding data formats such as complex coherent encoding ${ }^{18}$. Since their first demonstration in silicon technology ${ }^{2}$, RRMs have also been used for phase encoding ${ }^{19}$ or as phase shifters embedded in linear modulators ${ }^{20,21}$. Implementations with novel phase shifters such as silicon-organic hybrid slot waveguides ${ }^{22}$ as well as device implementation in a bulk CMOS compatible process flow ${ }^{23}$ have proven them to be versatile and widely applicable devices.

Due to their small dimensions, the capacitance $(C)$ of RRMs is small compared to longer linear devices. Since the energy per bit associated with switching a lumped element load ${ }^{24}$ with a drive voltage $V$ is given by $C V^{2} / 4$, this directly results in a much reduced RF power consumption, down to a few $\mathrm{fJ} / \mathrm{bit}$ demonstrated so $\mathrm{far}^{25}$. While RRMs are also resonant devices with a narrow passband that need to be actively temperature stabilized, recent progress has been made in reducing the associated power consumption either by reducing the thermal sensitivity 


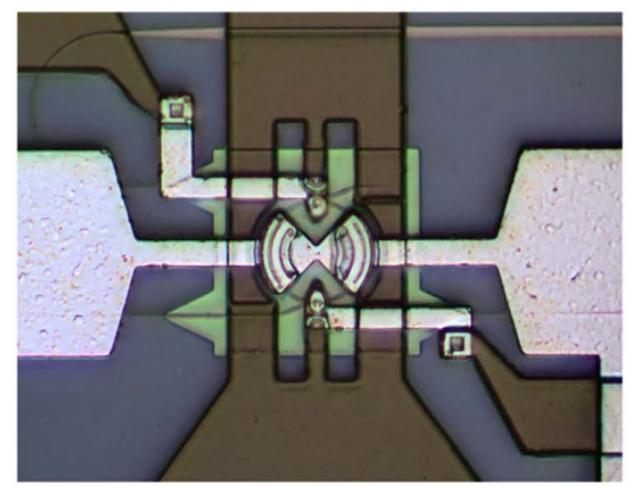

(a)

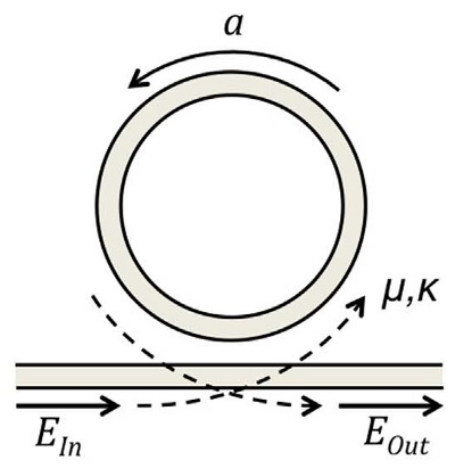

(b)

Figure $1 \mid$ Micrograph of the fabricated device. (a) Actual device, (b) Illustration of the symbols referred to in the text.

of the devices, e.g., by means of optical materials with opposing thermooptic coefficients ${ }^{26}$, or by increasing the power efficiency of the temperature tuning, e.g., by undercutting the structures ${ }^{27}$. With less than $100 \mathrm{fJ} / \mathrm{bit}$ added to the RF power consumption at $25 \mathrm{~Gb} / \mathrm{s}$ in ref. 27, the energy efficiency of RRMs approaches that of much more exotic and challenging to integrate devices such as hybrid plasmonicpolymer phase modulators ${ }^{28}$.

The RF power enhancement can be predicted by a simple metric, the finesse $F$ of the cavity given by the ratio of the free spectral range (the spectral distance between adjacent resonances) with the linewidth of the cavity. A reduced linewidth, and thus a higher quality factor (Q-factor), reduce the refractive index modulation required to achieve a given optical signal level. A smaller cavity, and thus a larger free spectral range, reduce the capacitance that has to be driven by the voltage source. It can be simply derived that a critically coupled RRM with a finesse $F$ has $1.3 F / \pi$ the power efficiency of a short lumped element modulator. Thus, while the latter can reach essentially the same small signal response (within a factor 2, see supplementary information), a RRM can be much more power efficient. Very small RRMs have been realized, with recent results reaching cavities as small as a few microns across ${ }^{25,29}$. The Q-factor on the other hand is typically limited by the required bandwidth of the device, so that electro-optic modulation efficiency and modulation bandwidth can be traded off against each other by statically or dynamically adjusting the Q-factor ${ }^{30,31}$.

Novel RRM configurations, for example with travelling wave electrodes $^{32}$ or with modulation of the coupling strength to the bus waveguide $^{33}$ have been proposed in order to increase the allowable Q-factor at high data rates. So far, however, lumped-element RRMs with resonant wavelength modulation (fig. 1) have found the widest reaching applicability. In these devices, the maximum Q-factor, and thus the resonant enhancement factor, are limited by the required E/ O bandwidth. Simplified models assume it to be independently limited by two factors: The RC time constant of the phase shifter, resulting in one first order filter, and the lifetime $\tau_{a}$ of photons in the cavity, resulting in a second first order filter with a bandwidth $1 / 2 \pi \tau_{a}$. The latter limits the maximum Q-factor that can be implemented, and thus ultimately the highest resonant enhancement that can be reached. Peaking in the transient modulator response arising from more complex time dynamics in the optical domain has been observed in several experimental studies $s^{6,9,12,34,35}$ with models of varying levels of complexity reported in the literature ${ }^{36-38}$. Here, we show that this transient response can be used to extend the frequency range of operation of RRMs beyond the cutoff predicted by the photon lifetime, extending the range of operation to higher data rates or, conversely, increasing the allowable Q-factor and reducing the RF power consumption. While this bandwidth improvement also comes at the cost of a reduced DC modulation efficiency, it provides an alternate and simpler path to reconfiguring optical transmitters. Moreover, a moderate improvement of the overall device performance can be achieved ${ }^{35}$.

We apply a first order perturbation theoretical treatment to the differential equations used to describe the time dynamics in $^{36,38}$ and derive a closed form expression of the frequency dependent small signal response of the modulator (E/O S-parameter) that matches very closely our measurements (fig. 2). Predicted asymmetric sideband generation is also verified experimentally (fig. 3), further confirming the model. After a theoretical investigation of peaking based enhancement (fig. 4), experimental characterization of optical links incorporating the RRM at 32, 40 and 44 Gbps demonstrates the capability of RRM peaking enhancement to enable high-speed optical links and to compensate for intersymbol interference (ISI)

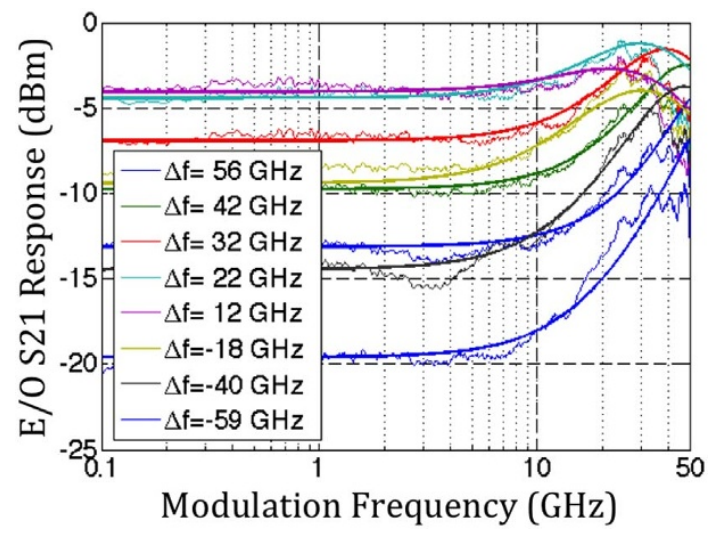

Figure $2 \mid$ Recorded and predicted E/O S-parameters. The E/O S21 of the RRM were recorded for different laser detunings (labeled in the legend) and compared to the model given by equation (3). Excellent agreement is seen up to $35 \mathrm{GHz}$ modulation frequency (beyond $35 \mathrm{GHz}$, back reflections from the probe tips at the RF output port of the chip have a significant impact on the recorded S21). Note that in the complete absence of dynamic waveguide losses, detuning the laser in either direction from the resonance would result in identical S21. The asymmetry of the two families of curves is adequately modeled by taking into account dynamic losses necessarily arising from the modulation of the free carrier density. Measurements were taken with $2 \mathrm{Vpp}$ and include DC photoreceiver responsivity and DC transimpedance amplifier gain (but with the frequency dependence of the receiver responsivity and cable losses deembedded). It should be noted that this ring was fabricated with implants optimized for travelling wave Mach-Zehnder modulators (as opposed to the results published in ref. 35 ) so that $2 \mathrm{Vpp}$ resulted in a small resonance shift of $5.5 \%$ of the resonance full width at half maximum (FWHM), well within the small signal regime. 

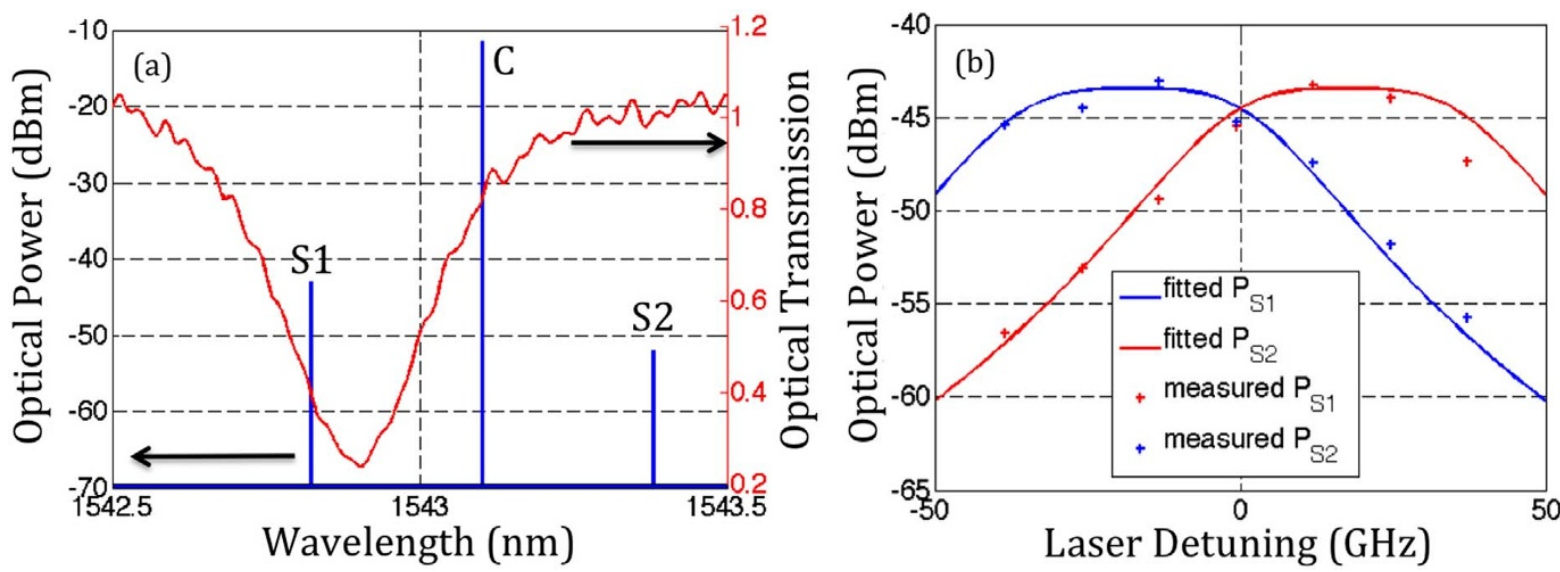

Figure 3 Recorded and predicted side bands generated by $35 \mathrm{GHz}$ RF modulation at different laser detunings. (a) shows an example of spectrum recorded with a high-resolution OSA at a laser detuning of $-24.6 \mathrm{GHz}$. The carrier (C) and two asymmetric sidebands (S1 and S2) are clearly visible. The plot is overlaid with the optical transmission spectrum of the ring to illustrate that the enhanced sideband is closer to the resonance. (b) Summary of the power generated in the sidebands as a function of laser detuning. Modulation with a detuned RRM results in asymmetric side bands adequately modeled by equation (4).

occurring elsewhere in the link (fig. 5). In order to make it straightforward for system architects not familiar with the intricacies of RRMs to leverage the peaking in communication systems, we show the modulator response to be equivalent to an amplifier with inductive peaking enhancement commonly used in high-speed communication architectures (fig. 6).

\section{Results}

Analytical modeling and experimental verification of peaking and asymmetric sideband generation. The investigated RRM (fig. 1) has a radius of $10.3 \mu \mathrm{m}$ and is constituted out of a $400 \mathrm{~nm}$ wide ridge waveguide partially etched into the $220 \mathrm{~nm}$ thick silicon device layer of a silicon-on-insulator wafer with a remaining silicon slab height of $90 \mathrm{~nm}$ around the waveguide. A reversed biased PIN junction with nominal implant concentrations $\mathrm{p}^{-}=3 \mathrm{e} 17 \mathrm{~cm}^{-3}$ and $\mathrm{n}^{-}=$ $1 \mathrm{e} 17 \mathrm{~cm}^{-3}$ overlapping by $50 \mathrm{~nm}$ serves as phase shifter. Electrical connectivity is obtained via highly doped regions with $\mathrm{p}^{+}$and $\mathrm{n}^{+}=$ $1 \mathrm{e} 20 \mathrm{~cm}^{-3}$. The total junction capacitance is $17 \mathrm{fF}$ at $-1 \mathrm{~V}$ bias and the series resistance $215 \Omega$, resulting in an electrically limited RC-bandwidth of $43 \mathrm{GHz}$ (details of the phase shifter can be found in ref. 11).

In order to derive the optical response of the modulator, we start with the equations introduced in refs. 36,38

$$
\begin{gathered}
\frac{\partial a}{\partial t}=\left(-i \omega_{r}-\frac{1}{\tau_{a}}\right) a+i \mu E_{I n} \\
E_{\text {Out }}=E_{\text {In }}+i \mu a
\end{gathered}
$$

where $a$ is the amplitude of the field travelling inside the resonator, $\omega_{r}$ the (angular) resonant frequency of the resonator, $\tau_{a}$ the $1 / \mathrm{e}$ decay time of the field amplitude (including both waveguide and coupling losses), $\mu$ the coupling strength between the resonator and the bus waveguide and $t$ the time. $E_{I n}$ and $E_{\text {Out }}$ are the amplitude of the waveguide mode incoming towards and outgoing from the modulator (fig. 1(b)). The time-domain coupling parameter $\mu$ verifies $\mu^{2}=$ $\kappa^{2} v_{\mathrm{g}} / L$, where $\kappa$ is the waveguide coupling parameter at the junction to the resonator, $v_{g}$ the group velocity of the waveguide constituting the ring and $L$ its circumference. By applying the sinusoidal drive voltage $\delta V \cdot \sin \left(\omega_{m} t\right)$ the resonant frequency of the resonator is shifted to $\omega_{r}+\delta \omega_{r} \cdot \sin \left(\omega_{m} t\right)$, where the nonlinearity of the transfer function is neglected since we are deriving the small signal S-parameter. We do assume however that $\delta \omega_{r}$ is a complex number with the imaginary part taking into account the modulation of the free-carrier induced losses that necessarily accompanies the index modulation ${ }^{39}$. The inclusion of this effect is necessary to describe the asymmetry of the recorded S21 for laser detuning to the left versus to the right of the ring resonance (fig. 2).

Equation (1) implies that a resonant frequency shift applied to the resonator is tracked by the light stored inside the resonator, i.e., preexisting light inside the resonator oscillates within the latter at its natural resonance frequency in the absence of additional light coupled from the waveguide (in analogy to a driven harmonic oscillator with damping ratio $1 / \tau_{a} \omega_{r}$, the incoming optical carrier playing the role of the driving force). The corresponding wavelength change is allowed by the broken time invariance of the externally driven resonator $^{40}$, an effect that has been experimentally verified ${ }^{41}$. By assuming $\delta V$ and $\delta \omega_{r}$ to be small quantities and applying first order perturbation theory (complete derivation in the supplementary information) we derive the small signal response of the modulator to be

$$
S_{21, \mathrm{EO}}=\mu \operatorname{Re}\left(\left[\frac{\delta \omega_{r} \bar{a}\left(\overline{E_{I n}}+i \mu \bar{a}\right)^{*}}{\frac{1}{\tau_{a}}-i \omega_{m}-\left(i \omega_{0}-i \omega_{r}\right)}+\frac{\delta \omega_{r}{ }^{*} \bar{a}^{*}\left(\overline{E_{I n}}+i \mu \bar{a}\right)}{\frac{1}{\tau_{a}}-i \omega_{m}+\left(i \omega_{0}-i \omega_{r}\right)}\right] e^{-i \omega_{m} t}\right)
$$

where $S_{21 \text {,EO }}$ is the electro-optic S21 (on a linear scale), $\omega_{0}$ is the frequency of the continuous wave optical carrier incoming from

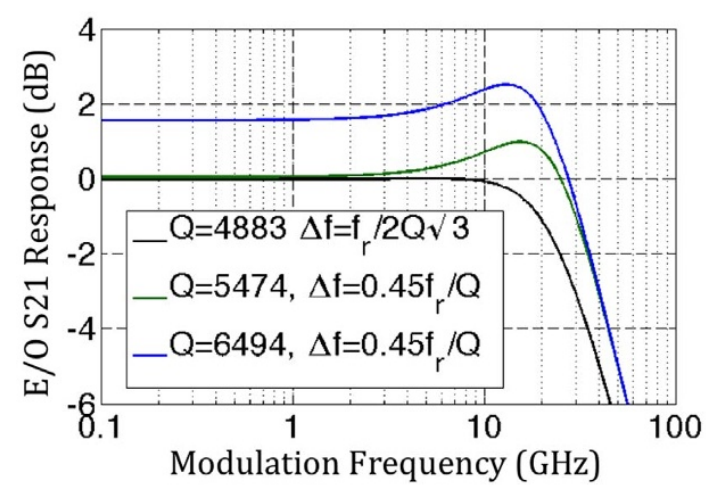

Figure $4 \mid$ Comparison between calculated S-parameters for different Qfactors and laser detunings. The black curve corresponds to the laser detuning expected to be the optimum based on a quasi-static analysis of the RRM, while the green and blue curves correspond to enhancement of either the bandwidth (green curve) or the modulation efficiency (blue curve) reoptimized taking peaking into account. All curves are normalized relative to the DC modulation efficiency of the black curve. Assumed Qfactors and laser detunings are indicated in the legend. 


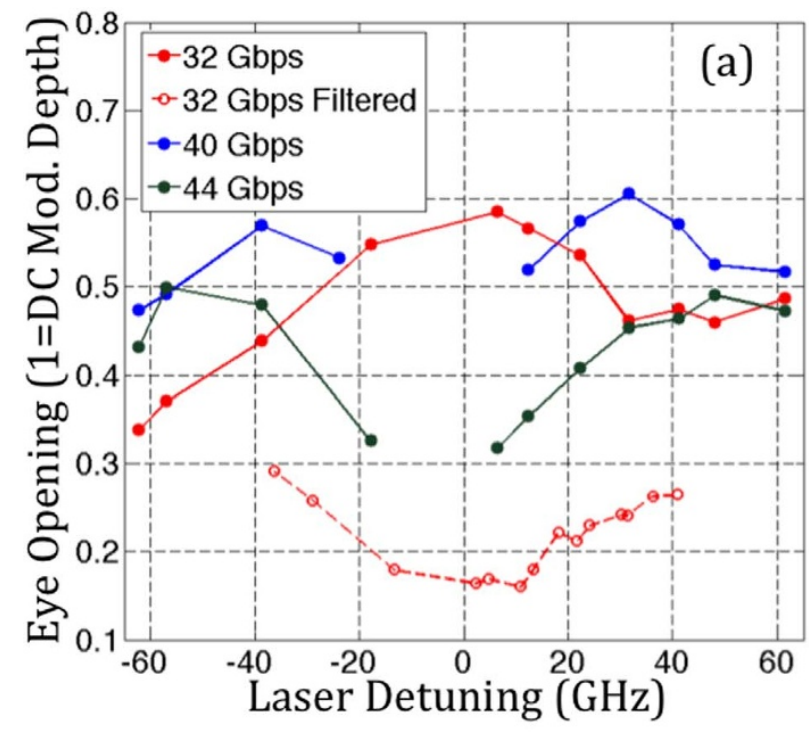

(c) 32 Gbps, Baseline 11.5 GHz Detuning

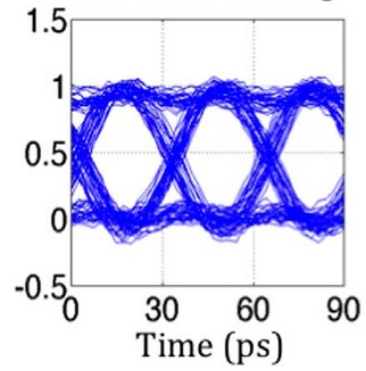

(d) 32 Gbps, Baseline $-33 \mathrm{GHz}$ Detuning

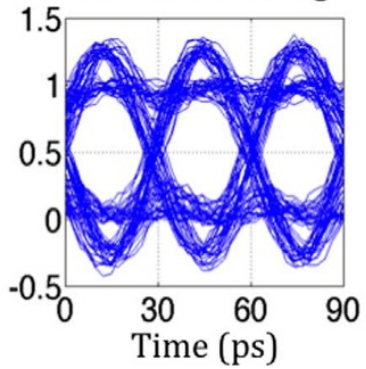

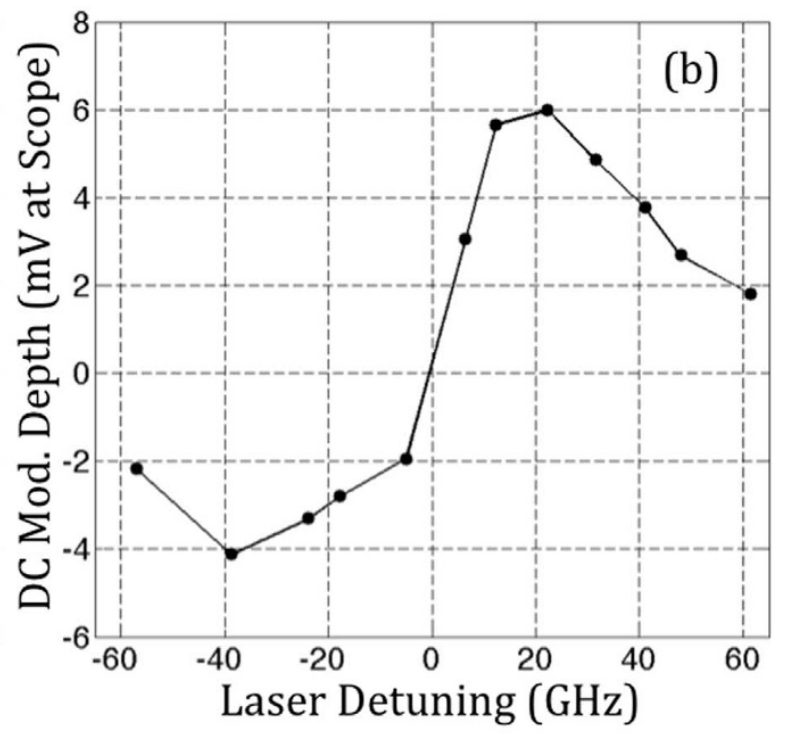

(e) $32 \mathrm{Gbps}$, Filtered 11.5 GHz Detuning

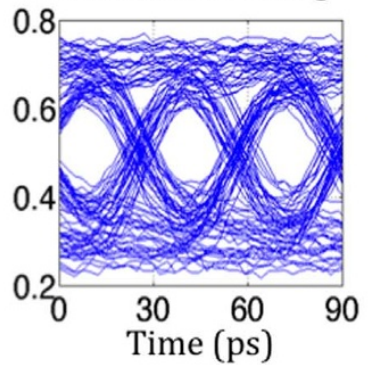

(f) 32 Gbps, Filtered $-33 \mathrm{GHz}$ Detuning

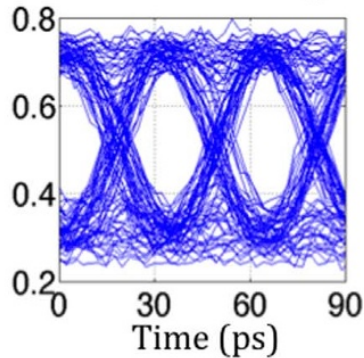

Figure $5 \mid$ Recorded RRM eye diagrams evidencing peaking enhancement. (a) Eye openings for different laser detunings at 32, 40 and 44 Gbps. At 32 Gbps, a second dataset was taken with additional penalization of the link ("filtered link") introduced by a long, low grade SMA cable ( $-8 \mathrm{~dB}$ attenuation at $16 \mathrm{GHz}$ modulation frequency). Eye openings are normalized to unit DC modulation efficiency. (b) summarizes the recorded DC modulation efficiency at different laser detunings. (c-f) show examples of individual eye diagrams in a penalized and non-penalized 32 Gbps link at low laser detuning (and thus small peaking) and moderate laser detuning (and high peaking). Here too, signal levels are normalized to unit DC modulation efficiency. Eye diagrams were recorded with PRBS 7 signals and 2 Vpp. Maximum signal levels in (e-f) are below one since the longest strings of consecutive zeros and ones were too short to fully reach stabilized DC levels. Eye diagrams are pattern lock averaged with a trigger generated every time the PRBS sequence is restarted, correctly representing ISI but averaging out receiver noise extrinsic to the RRM.

the laser, $E_{I n}=\overline{E_{I n}} e^{-i \omega_{0} t}$, and $\bar{a} e^{-i \omega_{0} t}$ is the value of $a$ in the absence of modulation voltage, i.e., its average or DC value $\bar{a}=\left\langle a \cdot \exp \left(i \omega_{0} t\right)\right\rangle$.

Measured E/O S21 were fitted with equation (3) for a complete set of laser wavelengths spanning the entire resonance linewidth of the

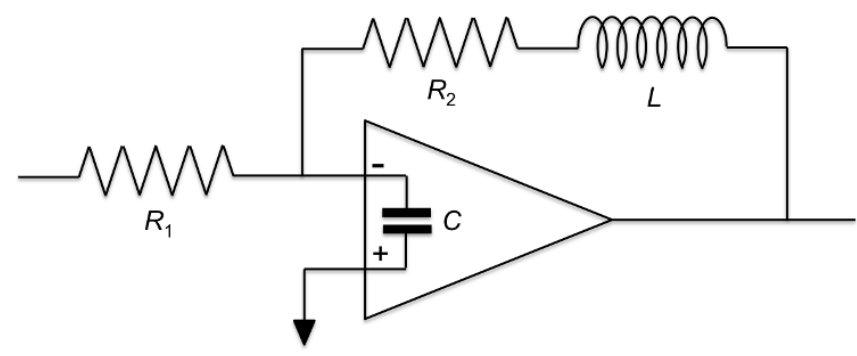

Figure $6 \mid$ Circuit diagram of an idealized peaking amplifier with identical transfer function as the RRM. Corresponding values of the lumped electrical elements for the transfer functions shown in fig. 2 are given in the supplementary information. Since peaking amplifiers are a commonly used building block in high-speed communication systems, this equivalent circuit makes the characteristics of the RRMs directly accessible to system architects. ring, with an excellent agreement between theory and experiment (fig. 2). The fitted change of photon lifetime versus resonance shift $\vartheta=\partial \tau_{a}{ }^{-1} / \partial \operatorname{Re}\left(\delta \omega_{r}\right)=-\partial \operatorname{Im}\left(\delta \omega_{r}\right) / \partial \operatorname{Re}\left(\delta \omega_{r}\right), 0.13$, is higher $(2 \times)$ but still reasonably close to the calculated value based on free carrier absorption and refractive index shift coefficients reported in the literature for bulk silicon ${ }^{39,42}$ (supplementary information). The Q-factors (3500 to 5000 depending on the wavelength) and waveguide coupling strengths were extracted from the experimental data. The laser detuning had to be corrected for thermal effects, i.e., it corresponds to the detuning between the frequency of the laser and the resonance of the ring as thermally shifted at the specific laser power and frequency. This was determined by comparing the thermally distorted optical transfer function of the ring to a transfer function recorded at low optical power featuring a near-perfect lorentzian line shape and mapping the points with corresponding extinction (see methods section). With the exception of a residual laser detuning below a few picometers due to drift during the measurements and of $\vartheta$, there were no fitted parameters. A description of the de-embedding procedure used to calibrate the S21 curves for RF losses occurring outside of the device can be found in the methods section.

The two additive terms of equation (3) correspond to the generation of two side lobes with asymmetric magnitudes 


$$
\delta a=-i \frac{\delta \omega_{r} \bar{a}}{2}\left(\frac{1}{i \omega_{r}-i \omega_{0}+i \omega_{m}+\frac{1}{\tau_{a}}} e^{\left(-i \omega_{0}+i \omega_{m}\right) t}+\frac{1}{i \omega_{r}-i \omega_{0}-i \omega_{m}+\frac{1}{\tau_{a}}} e^{\left(-i \omega_{0}-i \omega_{m}\right) t}\right)
$$

a prediction that was also confirmed by experiment and fitted with the same methodology as the E/O S21 (fig. 3).

Peaking occurs when the modulation frequency $\omega_{m}$ is close to the detuning of the laser frequency $\omega_{0}$ relative to the cavity resonance $\omega_{r}$, i.e., $\omega_{m}= \pm\left(\omega_{r}-\omega_{0}\right)$. Of the two sidebands, the sideband generated closest to resonance is also asymmetrically enhanced under these conditions. Peaking becomes more pronounced at large laser detuning on the order of or larger than $1 / 2 \pi \tau_{a}$, since the peaking frequency is then sufficiently far from DC for the peak to be resolved. A physical explanation in terms of time constants is given in the following.

As pointed out before ${ }^{12}$, overshoot in optical RRMs is the result of an interference effect between the light inside the modulator and the incoming light from the waveguide with a beating frequency given by the laser detuning. A perturbation theoretical treatment of the problem gives further insight into the physical mechanism: The response of the optical amplitude inside the ring resonator, $\delta a$, to the resonant frequency shift, $\delta \omega_{r}$, verifies

$$
\frac{\partial \delta a}{\partial t}=\left(-i \omega_{r}-\frac{1}{\tau_{a}}\right) \delta a-i \delta \omega_{r}(t) a
$$

In other words, the resonance frequency shift acts as a source term for $\delta a$ after which the generated perturbation continues to oscillate at the natural resonator frequency $\omega_{r}$ rather than the frequency of the incoming optical carrier $\omega_{0}$ (in the previously introduced analogy of a driven oscillator, the additional oscillation generated by a sudden perturbation $\delta \omega_{r}$ continues to oscillate at the natural frequency of the oscillator rather than the frequency of the driving force). Close to resonance, the light propagating inside the resonator normally couples back to the bus waveguide with a destructive interference relative to the incoming light, leading to the extinction seen at resonance in the optical transfer function of the $\mathrm{RRM}^{43}$ (the destructive interference can e.g. be derived from the fact that the coupling parameter between ring and waveguide is in quadrature to the junction transmission coefficient so that two coupling events from the waveguide to the resonator and back result in a -1 sign). A positive step perturbation $\delta \omega_{r}$ results in $\delta a$ being generated with a $\pi / 2$ phase advance relative to $a\left(\right.$ term $-i \delta \omega_{r}(t) a$ in equation (5)). $\delta a$ then continues to oscillate at the natural resonator frequency $\omega_{r}$ with a detuning $\omega_{r}-$ $\omega_{0}$ relative to the optical carrier. After a time delay $\pi / 2\left(\omega_{r}-\omega_{0}\right)$, an additional $\pi / 2$ phase advance is accumulated and the destructive interference condition converts into a constructive one, i.e., $\delta a$ couples constructively to the waveguide, resulting in an excess power coupled out of the cavity and an overshoot in the modulator response. A prerequisite for this to occur is of course that the perturbation $\delta a$ has not yet decayed, i.e., the time for the dephasing to occur, $\pi / 2\left(\omega_{r}-\omega_{0}\right)$, has to be shorter than the photon lifetime inside the cavity $\left(\tau_{a}\right)$. The overshoot results in an enhancement of the modulator response (S21) at RF frequencies close to $\omega_{r}-\omega_{0}$ : If one considers for example a square wave electrical drive signal with a period $\omega_{r}-\omega_{0}$, the overshoot occurs exactly halfway between the falling edge (rising $\delta \omega_{r}$ ) and the rising edge (falling $\delta \omega_{r}$ ), boosting the electrical " 0 "-level (optical "1"-level due to data inversion when $\omega_{r}$ $>\omega_{0}$ ). The same reasoning can be applied for a laser detuning corresponding to a negative $\omega_{r}-\omega_{0}$, in which case $\delta a$ is generated with a $\pi / 2$ phase retardation for a negative step perturbation $\delta \omega_{r}$ (rising edge of the electrical square wave) that is also converted to a $\pi$ dephasing after an additional $\pi / 2\left(\omega_{r}-\omega_{0}\right)$ time delay, boosting the electrical "1"-level (also optical "1"-level). In summary, the interference between $a$ and $\delta a$ results in ringing with a frequency $\left|\omega_{0}-\omega_{r}\right|$ in the response of the RRM to a short pulse, so that periodic driving of the RRM at a frequency $\omega_{m}=\left|\omega_{0}-\omega_{r}\right|$ synchronized with the impulse response of the ringing leads to an enhanced S21 (peaking).
Application to ISI reduction in ultra-high-speed optical links. The peaking predicted and experimentally verified in the previous section can be used to enhance the characteristics of RRMs, with the possibility of either enhancing the bandwidth of the device or its drive voltage/RF power consumption depending on the priorities driven by the system architecture. Numerical examples are first given based on the model derived in the previous section. The effect is then experimentally verified with measured eye diagrams.

At low frequencies, the maximum response of a critically coupled RRM is obtained for a laser detuning of $\Delta \omega=1 / \sqrt{3} \tau_{a}$ (see supplementary information). Further detuning of the laser results in a reduction of the DC response, but also results in an extended bandwidth via peaking and can increase the eye opening at high data rates. The optimum laser detuning depends on the data rate and can be deduced from equation (3) together with the S-parameters of the rest of the system. In order to exemplify this, we calculated E/O Sparameters for different Q-factors and laser detunings that illustrate how either power consumption, drive voltage or bandwidth can be improved via the RRM peaking.

Figure 4 shows a comparison between E/O S-parameters calculated for different $\mathrm{Q}$-factors and laser detunings. The other parameters are identical to the ones used to model the experimental results in fig. 2 (in particular, the phase tuner's phase shift is assumed to remain unchanged and critical coupling is assumed to be maintained). The black curve corresponds to a laser detuning of $\Delta \omega=\omega_{r} / 2 \sqrt{3} Q$ resulting in the highest low speed modulation efficiency given the assumed Q-factor (4883) and no noticeable peaking. The green curve corresponds to an increased Q-factor (5474) with the resulting decrease in $\mathrm{E} / \mathrm{O}$ bandwidth overcompensated by a larger laser detuning $\Delta \omega=0.45 \omega_{r} / Q$ and a stronger peaking. The DC modulation efficiency is the same (the higher Q-factor is compensated by the larger detuning), but the bandwidth is increased from $30 \mathrm{GHz}$ to $40 \mathrm{GHz}$ when referenced to the $-3 \mathrm{~dB}$ level below the DC S21 and to $35 \mathrm{GHz}$ when referenced to $-3 \mathrm{~dB}$ level below the peak of the S21 curve. The blue curve also corresponds to $\Delta \omega=0.45 \omega_{r} / Q$, with a Q-factor further increased to 6494. The E/O bandwidth drops back to its initial level $(33 \mathrm{GHz}$ when referenced to the DC S21, $29 \mathrm{GHz}$ when referenced to the peak of the S21), but the DC S21 is enhanced by $1.56 \mathrm{~dB}$ (defined as $20 \log 10(\mathrm{OMA})$ ). It should be noted that referencing the $3 \mathrm{~dB}$ roll-off to the peak of the S21 is the more conservative and fairer metric, since peaking also introduces phase distortion. It can be concluded from these curves that optimizing the laser detuning for maximum DC modulation amplitude, as would be the natural choice when peaking is ignored, is suboptimal in either case. A systematic experimental study of the modulation efficiency/ modulation bandwidth trade-off based on equation (3) can be found in ref. 35, albeit critical coupling was not maintained.

Figure 5 shows examples of eye diagrams and summarizes eye openings recorded with the investigated RRM at different laser detunings and data rates, exemplifying the benefits of peaking in complete optical links. The baseline 32 Gbps link summarized by the solid red curve in fig. 5(a) is far from being bandwidth limited and the transient ringing corresponding to the S-parameter peaking in the time domain partially closes the eyes instead of opening them. At higher data rates, however, the optical links are bandwidth limited and the peaking contributes to extending the bandwidth of the link and reopening the eyes. At $40 \mathrm{Gbps}$, the eyes could for example be reopened to the same level as in the baseline $32 \mathrm{Gbps}$ link. The optimum peaking depends on the data rate, with the optimum laser detuning increasing at higher data rates. Similarly, the peaking can also contribute to recovering good performance in a link that is heavily bandwidth limited due to other components. To exemplify this, we recorded eye diagrams after penalizing the 32 Gbps link with an additional low grade SMA cable $(-8 \mathrm{~dB}$ attenuation at the Nyquist frequency, $16 \mathrm{GHz}$ ). A substantial peaking enhancement 


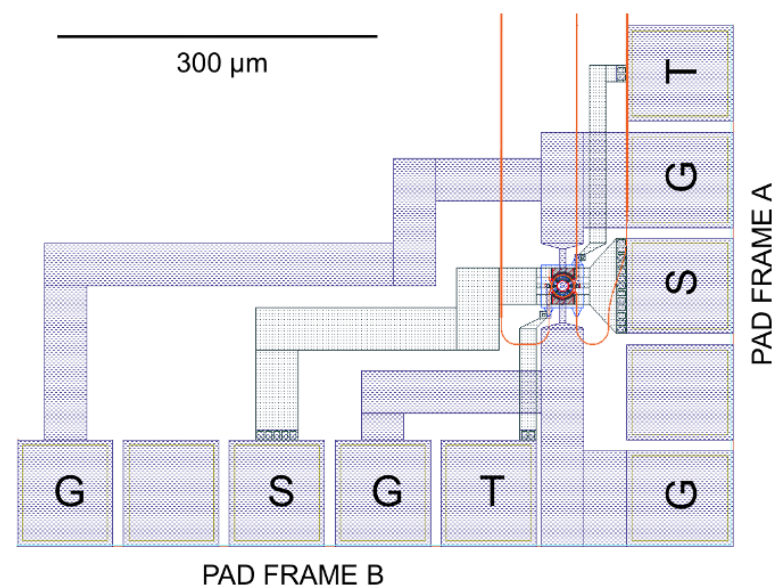

PAD FRAME B

Figure 7 Snapshot of the mask definition. Pad frames A and B referenced in the text are marked. Pads labeled by G, S and T are respectively ground pads, signal pads and pads used to apply a control signal to thermally tune the rings. Waveguides are shown in orange.

of the optical link then also becomes visible. Figures 5(c)-(f) are examples of 32 Gbps eye diagrams with and without extrinsic penalization, as well as with low and high peaking. In case of the baseline 32 Gbps link, peaking is clearly visible in the form of an overshoot of rapidly changing data patterns (fig. $5(\mathrm{~d})$ ), while in the penalized 32 Gbps optical link peaking is visible in the form of a reopening of the eye diagram (fig. 5(f)). A net enhancement of the absolute OMA cannot be observed in this dataset (i.e., the product of the data shown in figs. 5(a) and 5(b)) because even for the most highly penalized link shown in fig. 5 (dashed red curve in (a) and eye diagrams (e) and (f)), the eye diagram is already completely reopened at $-33 \mathrm{GHz}$ detuning (fig. 5(f)) making further bandwidth enhancement unnecessary and detuning beyond the DC OMA optimum detrimental. Such an effect is however expected to be apparent in more heavily filtered links or with higher Q-factor RRMs optimized for lower speeds.

In order to leverage the peaking in a communication system architecture, it is useful to describe the response of the RRM with a wellknown equivalent electric circuit, facilitating the work for system architects not familiar with the intricacies of this device. The idealized peaking amplifier shown in fig. 6 actually has exactly the same transfer function for adequately chosen electrical components. Equation (3) can be recast into

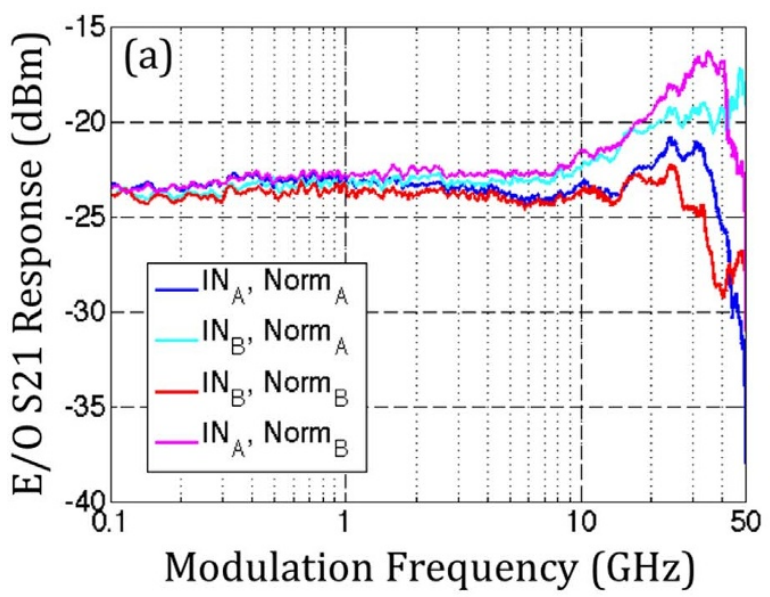

$$
S_{21, \mathrm{EO}}=\operatorname{Re}\left(\frac{2 \operatorname{Re}(\eta) \frac{1}{\tau_{a}}-2 \operatorname{Im}(\eta) \Delta \omega-2 \operatorname{Re}(\eta) i \omega_{m}}{\frac{1}{\tau_{a}{ }^{2}}+\Delta \omega^{2}-2 i \frac{\omega_{m}}{\tau_{a}}-\omega_{m}{ }^{2}} e^{-i \omega_{m} t}\right)
$$

where $\eta=\mu \delta \omega_{r} \bar{a}\left(\overline{E_{I n}}+i \mu \bar{a}\right)^{*}$ and $\Delta \omega=\omega_{0}-\omega_{r}$ is the laser detuning. This then takes the same form as the transfer function of a peaking amplifier

$\frac{V_{\text {Out }}}{V_{\text {In }}}=\operatorname{Re}\left(-\frac{A_{v}\left(R_{2}-i L \omega_{m}\right)}{R 1\left(A_{v}+1\right)+R_{2}-i L \omega_{m}-i C R_{1} R_{2} \omega_{m}-C L R_{1} \omega_{m}^{2}} e^{-i \omega_{m} t}\right)$

where $V_{\text {In }}$ and $V_{\text {Out }}$ are the input and output voltages of the amplifier, $R_{1}, R_{2}, C$ and $L$ are the lumped element electrical components shown in fig. 6 and $A_{v}$ is the voltage gain of the operational amplifier (note that equation (7) is complex conjugated compared to the usual convention used in electronics to make it consistent with the convention for time dependence as $\exp (-i \omega t)$ used here and elsewhere in the paper).

\section{Discussion}

We have modeled the transient E/O response of RRMs with a closed form expression derived with perturbation theory that predicts both peaking in the S-parameter as well as asymmetrically generated side bands. The de-embedding methodology developed for the characterization of the RRMs allows the determination of the E/O response of the RRM with sufficient precision to enable a one to one comparison with the theoretical RRM response. Both predictions are verified experimentally with excellent agreement. The impact of peaking on ISI reduction in RRM based optical links is demonstrated based on the reopening of eye diagrams at high data rates as well as in transmission systems penalized elsewhere in the link. An equivalent circuit description of the RRM allows straightforward evaluation of this effect on optical datacom links with existing system analysis methodologies.

The results reported in this paper show that complex time dynamics need to be taken into account when interpreting measurement data and optimizing RRM designs even for the well-known lumped element RRMs relying on resonant frequency modulation. Simple models reducing the time dynamics of RRMs to a single pole filter resulting from the photon lifetime in the cavity fail to account for the actual response of the devices. In particular, leveraging peaking in a communication system architecture allows moderately increasing the quality factor of the RRMs without compromising data integrity, thus reducing the drive voltage and the power consumption and is thus an important aspect for device performance.

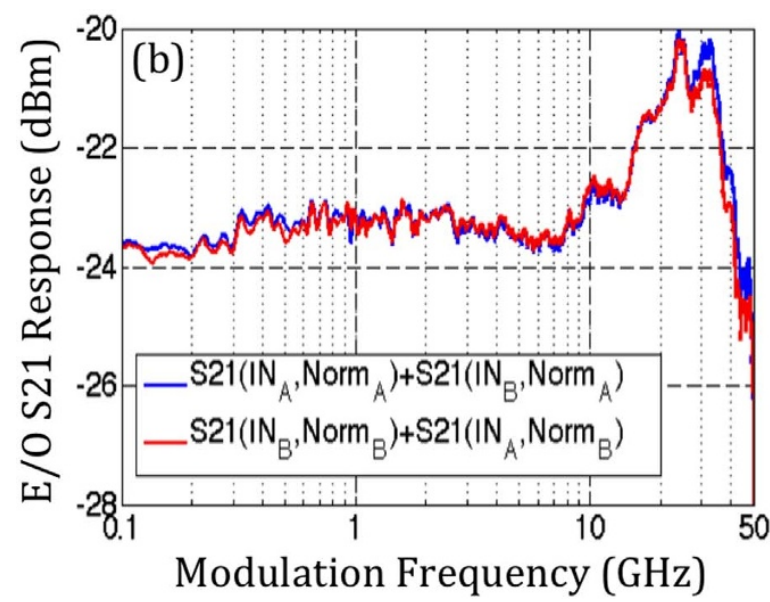

Figure 8 Example of datasets taken with different test setup configurations and resulting de-embedded S-parameter. (a) shows the four measurement configurations, with electrical signals injected either through pad frame $\mathrm{A}$ or $\mathrm{B}\left(\mathrm{IN}_{\mathrm{A}}\right.$ and $\left.\mathrm{IN}_{\mathrm{B}}\right)$ and $\mathrm{S} 21$ curves normalized relative to the signal levels at either pad frame $\left(\right.$ Norm $_{\mathrm{A}}$ and $\left.\mathrm{Norm}_{\mathrm{B}}\right)$. (b) shows the averaged S-parameters obtained with two different configurations, with excellent consistency obtained between the two datasets. 

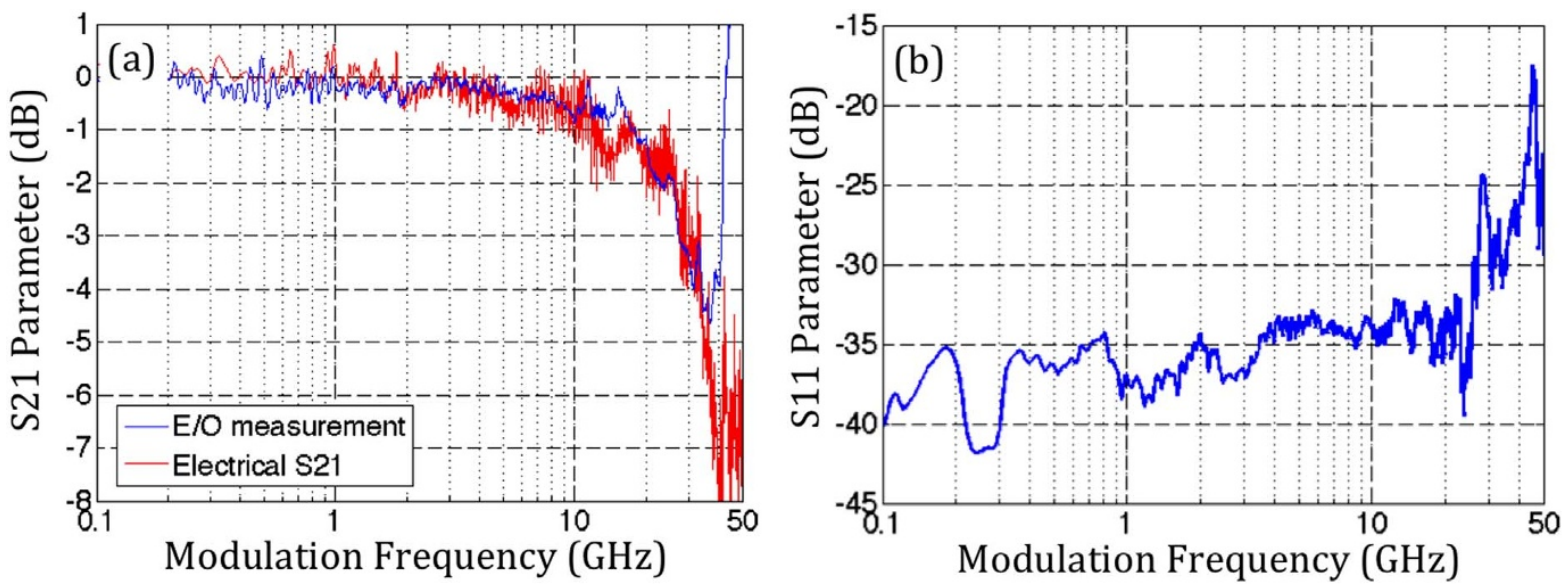

Figure $9 \mid$ Comparison between the on-chip losses extracted from the E/O measurements to a direct measurement of the electrical S21 of the chip (a) and measured back-reflections at the chip input (b). We attribute the strong increase in the dataset extracted from the difference of measured E/O responses in (a) to the increased RF back-reflections seen in (b) above $35 \mathrm{GHz}$, as explained in the text.

Given the relative simplicity of the closed form expression derived for the E/O RRM response, taking RRM time dynamics into account in future communication system architectures should be greatly facilitated.

\section{Methods}

De-embedding of the E/O S21. The RRM is connected to two GSG pad frames, one serving as an input and one serving as a $50 \Omega$ terminated output, both being accessed with $40 \mathrm{GHz}$ rated probe tips. The entire setup was based on K-type connectors rated up to $46 \mathrm{GHz}$. Both the E/O S21 and the electrical S21 of the chip were measured; the latter defined as the transmission from the input pad frame to the output pad frame. The on-chip signal traces between either of the two pad frames and the RRM are of different lengths (fig. 7), resulting in the measured RRM bandwidth depending on the pad frame used as the input port. In addition to normalizing out the cable attenuation and the S-parameter of the receiver used in the test setup, following procedure was employed to de-embed the on-chip RF attenuation: First the device was measured by injecting the RF signal into pad frame A and normalizing the S21 relative to the electrical signal level arriving at pad frame A obtained by subtracting cable losses from the VNA output (source). This underestimates the S21 by the amount of electrical signal losses occurring between pad frame A and the modulator (S21(InA, NormA)). In a second measurement, the signal was injected into pad frame $B$, while pad frame A served as the $50 \Omega$ terminated chip output. The measurement was normalized by a different method, relative to the electrical signal level at the output pad frame of the chip obtained by normalizing out cable losses from the signal received at the VNA input (sink) used to measure the electrical S21. This overestimates the S21 by the same amount, i.e., the electrical signal losses between pad frame A and the modulator (S21(InB, NormA)). The de-embedded signal was then taken as the mean of the two datasets, since they respectively overestimate and underestimate the O/E S21 by the same amount (fig. 8). This method proved to be extremely reliable up to $35 \mathrm{GHz}$. Swapping the role of pad frames A and B in both measurements resulted in the same de-embedded S21. In addition, to validate the methodology, we compared the onchip signal losses extracted from O/E measurements S21(InA, NormA)-S21(InB, NormB) with the on-chip signal losses obtained directly from the electrical S21, with excellent consistency up to $35 \mathrm{GHz}$ (fig. 9(a)). Beyond this point, there is a sharp difference between the directly measured electrical signal loss and the dataset extracted from the $\mathrm{O} / \mathrm{E}$ measurements attributed to strong RF reflections at the chip output pads. These reflections were independently confirmed with the electrical S11 of the chip (fig. 9(b)). They lead to a sharp enhancement or penalization of the measured E/O S-parameter depending on which pad frame is used as an output, which is attributed to a RF node or antinode forming at the RRM depending on the distance to the pad frame. In particular, the additional drop-off relative to the predicted S21 does not follow the single pole dependency associated to the intrinsic electrical bandwidth of the modulator. It should also be noted that while this deembedding procedure may appear intricate, it is only a minor refinement: Pad frame A is much closer to the modulator than pad frame B, so that very small losses occur between these two elements. Taking pad frame A as an input and normalizing the S21 relative to the electrical signal level arriving at pad frame A results in almost the same S21, within $1.5 \mathrm{~dB}$ up to $35 \mathrm{GHz}$ (fig. 8). S-parameters were recorded with an Agilent $50 \mathrm{GHz}$ VNA (N5245A) and a U2T high-speed photoreceiver (40 GHz bandwidth XPRV2021A). Eye diagrams were generated with an Anritsu $56 \mathrm{G}$ pattern generator consisting of an MP1800 BERT System, 4 MU181020B 14 G pattern generators and an MP1821A MUX and recorded with a $50 \mathrm{GHz}$ sampling oscilloscope from Agilent (83484A). Sideband measurements were done with a high resolution OSA from APEX (AP2043B) resolving $5 \mathrm{MHz}$ optical frequency.

Figure 7 shows a snapshot of the chip layout. Two pad frames enable electrical connectivity to GSG signal lines, with one pad frame used to inject the signal from the VNA and the other to return the signal to the VNA, both $50 \Omega$ terminating the chip and allowing recording the electrical S21 of the chip. De-embedding the signal

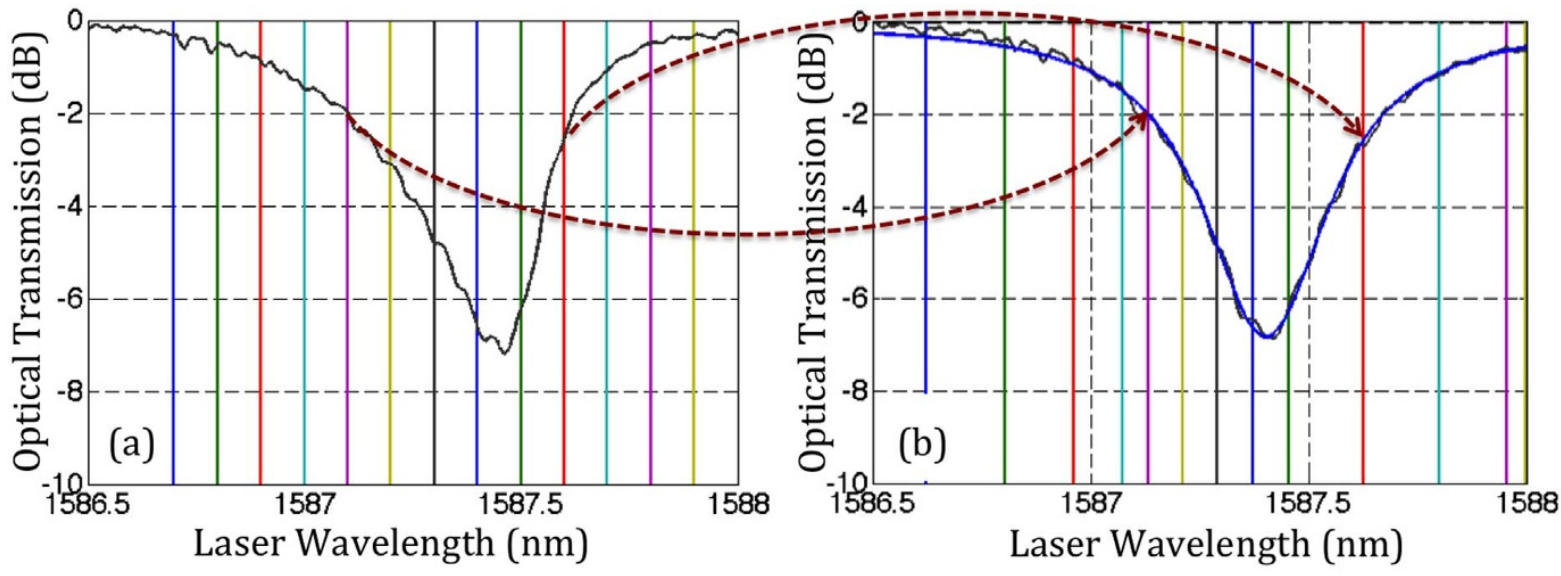

Figure $10 \mid$ Comparison of the ring transfer function recorded at high (10 dBm in waveguide) and low ( $-6 \mathrm{dBm}$ in waveguide) optical power levels and determination of the laser detuning relative to the thermally shifted resonant frequency. The laser detuning read from (b) after mapping is also the actual laser detuning relative to the thermally shifted resonance for (a) and is the detuning taken into account in the fitting of the experimental results. The transfer function in (b) is overlaid with a fit corresponding to an ideal ring model (i.e., without thermal effects). 
attenuation occurring in the SMA cables used in the test setup is straightforward since their S-parameters are directly accessible. Signal attenuation on the chip however also plays a role and is harder to de-embed. While measuring the electro-optic response of the RRM by injecting the electrical signal through the pad frame with the shorter signal lines (pad frame A) and taking into account the cable losses between the VNA and the chip input already results in quite accurate results (within $1.5 \mathrm{~dB}$ of the more accurate de-embedding procedure described here), we were able to obtain more accurate results by taking several measurements into account obtained with different test setup configurations.

Figure 8 shows examples of the four datasets corresponding to the different input pads and normalizations. As explained above, S21 $\left(\mathrm{In}_{\mathrm{A}}, \mathrm{Norm}_{\mathrm{A}}\right)$ underestimates the E/ O S-parameters by the amount of electrical attenuation between pad frame A and the RRM, while S21 $\left(\operatorname{In}_{B}, \mathrm{Norm}_{\mathrm{A}}\right)$ overestimates the E/O S-parameter by the same amount. Thus the average of the two curves is a good estimate of the de-embedded $\mathrm{E} /$ O S21. Normalizing relative to the signal levels at pad frame B increases the error, since the attenuation between the pad frame and the device is higher due to the longer signal traces. However, averaging the two curves $\mathrm{S} 21\left(\mathrm{In}_{\mathrm{B}}, \mathrm{Norm}_{\mathrm{B}}\right)$ and

S21 $\left(\operatorname{In}_{A}\right.$, Norm $\left._{B}\right)$ again averages out the opposing measurement biases and results in the same de-embedded curve than previously (fig. 8(b)). Consistency between these two procedures indicates that the de-embedding methodology is adequate.

In order to independently verify the adequacy of the de-embedding procedure, a simple test can be made consisting in comparing the on-chip losses extracted from the difference between raw E/O S-parameters taken by injecting the electrical signal into either pad frame A or pad frame B to the on-chip RF losses directly extracted from the electrical S21. The comparison between the two curves can be seen in fig. 9. It is apparent that up to $35 \mathrm{GHz}$ modulation frequency the consistency is excellent, confirming that up to this point on-chip signal distortion is primarily due to attenuation. At higher frequencies, there is a sharp discrepancy seen in the data extracted from the E/O S21.

This discrepancy is attributed to RF reflections induced at the pad frame or the probe tip at the output of the chip. Indeed, the reflected RF wave can add-up constructively or destructively to the forward propagating wave at the RRM, either boosting or reducing the observed S21. The effect of such a back-reflection is thus sensitive to the electrical path length between the output pad frame and the device. The reflections are further confirmed by directly measuring the electrical power returning to the VNA. As seen in fig. 9(b), S11 grows substantially above $35 \mathrm{GHz}$. It can be concluded from the entire dataset that the de-embedding procedure works extremely well up to $35 \mathrm{GHz}$ modulation frequency, after which RF reflections play a dominant role.

Thermal effects on the effective laser detuning. A second effect that needs to be carefully taken into account is the thermal resonance shift of the RRM induced by optical absorption in the ring. Since the amount of absorbed optical power, and thus the amount of self-heating and thermal shift, depends on the detuning of the laser, a separate corrective factor needs to be applied for every laser wavelength. The required corrective factors could be straightforwardly experimentally determined by comparing a ring resonance recorded under the same conditions as the $\mathrm{E} / \mathrm{O}$ responses (10 $\mathrm{dBm}$ in the waveguide) to the same resonance recorded at low optical power levels with negligible non-linearity ( $-6 \mathrm{dBm}$ in the waveguide). As is illustrated in fig. 10, points in the transfer functions with identical extinction are mapped to each other. The effective laser detuning is then taken as the detuning in the mapped diagram (dataset with a lorentzian line shape recorded at low optical power levels). Since thermal effects are too slow to track the ring dynamics at the recorded modulation frequencies, the resonant frequency of the ring can be considered as being simply DC shifted during the RF measurements.

1. Rabiei, P., Steier, W. H., Zhang, C. \& Dalton, L. R. Polymer Micro-Ring Filters and Modulators. J. Lightwave Technol. 20, 1968 (2002).

2. Xu, Q., Schmidt, B., Pradhan, S. \& Lipson, M. Micrometer-scale silicon electrooptic modulator. Nat. 435, 325-327 (2005).

3. Dong, P. et al. Low $\mathrm{V}_{\mathrm{pp}}$, ultralow-energy, compact, high-speed silicon electrooptic modulator. Opt. Express 17, 22484-22490 (2009).

4. Gardes, F. Y. et al. High-speed modulation of a compact silicon ring resonator based on a reverse-biased pn diode. Opt. Express 17, 21986-21991 (2009).

5. Li, G. et al. $25 \mathrm{~Gb} / \mathrm{s} 1 \mathrm{~V}$-driving CMOS ring modulator with integrated thermal tuning. Opt. Express 19, 20435-20443 (2011).

6. Xiao, X. et al. 44-Gb/s Silicon Microring Modulators Based on Zigzag PN Junctions. Photon. Technol. Lett. 24, 1712-1714 (2012).

7. Arakawa, Y., Nakamura, T., Urino, Y. \& Fujita, T. Silicon Photonics for Next Generation Integration Platform. IEEE Commun. Mag. 51, 72-77 (2013).

8. Jalali, B. \& Fathpour, S. Silicon Photonics. J. Lightwave Technol. 24, 4600-4615 (2006).

9. Xu, Q., Manipatruni, S., Schmidt, B., Shakya, J. \& Lipson, M. 12.5 Gbit/s carrierinjection-based silicon micro-ring silicon modulators. Opt. Express 15, 430-436 (2007).

10. Thomson, D. J. et al. 50-Gb/s Silicon Optical Modulator. Photon. Technol. Lett. 24 234-236 (2012).

11. Merget, F. et al. Silicon photonics plasma-modulators with advanced transmission line design. Opt. Express 21, 19593-19607 (2013).
12. Xu, Q., Schmidt, B., Shakya, J. \& Lipson, M. Cascaded silicon micro-ring modulators for WDM optical interconnection. Opt. Express 14, 9431-9435 (2006).

13. Lelarge, F. et al. Recent Advances on InAs/InP Quantum Dash Based Semiconductor Lasers and Optical Amplifiers Operating at $1.55 \mu \mathrm{m}$. J. Select. Topics. Quantum Electron. 13, 111-124 (2007).

14. Gubenko, A. et al. Error-free $10 \mathrm{Gbit} / \mathrm{s}$ transmission using individual Fabry-Perot modes of low-noise quantum-dot laser. Electron. Lett. 43, 1430-1431 (2007).

15. Del'Haye, P. et al. Optical frequency comb generation from a monolithic microresonator. Nat. 450, 1214-1217 (2007).

16. Razzari, L. et al. CMOS-compatible integrated optical hyper-parametric oscillator. Nat. Photonics 4, 41-45 (2010).

17. Levy, J. S. et al. CMOS-compatible multi-wavelength oscillator for on-chip optical interconnects. Nat. Photonics 4, 37-40 (2010).

18. Pfeifle, J. et al. Coherent data transmission with microresonator Kerr frequency combs. Nat. Photonics 8, 375-380 (2014).

19. Padmaraju, K. et al. Error-free transmission of microring-modulated BPSK. Opt. Express 20, 8681-8688 (2012)

20. Xie, X., Khurgin, J., Kang, J. \& Chow, F.-S. Linearized Mach-Zehnder Intensity Modulator. Photon. Technol. Lett. 15, 531-533 (2003).

21. Gutierrez, A. M. et al. Ring-Assisted Mach-Zehnder Interferometer Silicon Modulator for Enhanced Performance. J. Lightwave Technol. 30, 9-14 (2012).

22. Gould, M. et al. Silicon-polymer hybrid slot waveguide ring-resonator modulator. Opt. Express 19, 3952-3961 (2011).

23. Shainline, M. J. et al. Depletion-mode polysilicon optical modulators in a bulk complementary metal-oxide semiconductor process. Opt. Lett. 38, 2729-2731 (2013).

24. Miller, D. A. B. Energy consumption in optical modulators for interconnects. Opt Express 20, A293-A308 (2012).

25. Watts, M. R., Zortman, W. A., Trotter, D. C., Young, R. W. \& Lentine, A. L. Vertical junction silicon microdisk modulators and switches. Opt. Express 19, 21989-22003 (2011).

26. Teng, J. et al. Athermal Silicon-on-insulator ring resonators by overlaying a polymer cladding on narrowed waveguides. Opt. Express 17, 14627-14633 (2009).

27. Dong, P. et al. M. Thermally tunable silicon racetrack resonators with ultralow tuning power. Opt. Express 18, 20298-20304 (2010).

28. Melikyan, A. et al. High-speed plasmonic phase modulators. Nat. Photonics 8 , 229-233 (2014).

29. Manipatruni, S., Preston, K., Chen, L. \& Lipson, M. Ultra-low voltage, ultra-small mode volume silicon microring modulator. Opt. Express 18, 18235-18242 (2010).

30. Gill, D. M. et al. Internal Bandwidth Equalization in a CMOS-Compatible Si-Ring Modulator. Photon. Technol. Lett. 21, 200-202 (2009).

31. Gill, D. M. et al. CMOS-Compatible Si-Ring-Assisted Mach-Zehnder Interferometer With Internal Bandwidth Equalization. J. Select. Topics Quantum Electron. 16, 45-52 (2010).

32. Bortnik, B. et al. Electrooptic polymer ring resonator modulation up to $165 \mathrm{GHz}$. J. Select. opics Quantum Electron. 13, 104-110 (2007).

33. Sacher, W. D. et al. Coupling modulation of microrings at rates beyond the linewidth limit. Opt. Express 21, 9722-9733 (2013).

34. Yu, H. et al. Trade-off between optical modulation amplitude bandwidth of silicon micro-ring modulators. Opt. Express 22, 15178-15189 (2014).

35. Sharif Azadeh, S. et al. Advances in Silicon Photonics Segmented Electrode MachZehnder Modulators and Peaking Enhanced Resonant Devices. Proc. SPIE 9288 (2014). In press.

36. Little, B. E., Chu, S. T., Haus, H. A., Foresi, J. \& Laine, J.-P. Microring Resonator Channel Dropping Filters. J. Lightwave Technol. 15, 998-1005 (1997).

37. Sacher, W. D. \& Poon, J. K. S. Dynamics of microring resonator modulators. Opt. Express 20, 15741-15753 (2008)

38. Zhang, L. et al. Silicon-based Microring Resonator Modulators for Intensity Modulation. J. Select. Topics Quantum Electron. 16, 149-158 (2010).

39. Soref, R. A. \& Bennett, B. R. Electrooptical Effects in Silicon. J. Quantum Electron. 23, 123-129 (1987)

40. Notomi, M. \& Mitsugi, S. Wavelength conversion via dynamic refractive index tuning of a cavity. Phys. Rev. A 73, 051803 1-4 (2006)

41. Preble, S. F., Xu, Q. \& Lipson, M. Changing the color of light in a silicon resonator Nat. Photonics 1, 293-296 (2007).

42. Lin, Q., Painter, O. J. \& Agrawal, G. P. Nonlinear optical phenomena in silicon waveguides: Modeling and applications. Opt. Express 15, 16604-16644 (2007).

43. Yariv, A. Critical Coupling and Its Control in Optical Waveguide-Ring Resnator Systems. Photon. Technol. Lett. 14, 483-485 (2002).

\section{Acknowledgments}

The authors gratefully acknowledge funding from the European Research Council (FP7/ 2011-2016 279770) for the project "Frontiers of Integrated Silicon Nanophotonics in Telecommunications" and from the European Commission under contracts 293767 and 619591 for the projects "Silicon Photonics Coherent Optical Wavelength Division Multiplexed Transceiver" and "Broadband Integrated and Green Photonic Interconnects for Higher-Performance Computing and Enterprise Systems". The chips were fabricated at the Singapore Institute of Microelectronics (IME) via the shuttle service OpSIS. 


\section{Author contributions}

J.W. conceived the experiments and derived the theory, F.M. and S.R.G. designed the device, J.M. and S.S.A. measured the device, J.M. and J.W. analyzed the data, J.H. and B.S. contributed to the test setup, J.W. wrote the paper.

\section{Additional information}

Supplementary information accompanies this paper at http://www.nature.com/ scientificreports

Competing financial interests: The authors declare no competing financial interests.
How to cite this article: Müller, J. et al. Optical Peaking Enhancement in High-Speed Ring Modulators. Sci. Rep. 4, 6310; DOI:10.1038/srep06310 (2014).

(1) This work is licensed under a Creative Commons Attribution-NonCommercialcc) article are included in the article's Creative Commons license, unless indicated otherwise in the credit line; if the material is not included under the Creative Commons license, users will need to obtain permission from the license holder in order to reproduce the material. To view a copy of this license, visit http:// creativecommons.org/licenses/by-nc-sa/4.0/ 